\title{
Alte und neue Herausforderungen einer sozial gerechten Bildungspolitik
}

\author{
In den letzten Jahren sind in Deutschland viele Bildungsreformen umgesetzt \\ worden, die in der Grundtendenz aus einer Perspektive der sozialen Gerechtigkeit \\ positiv zu bewerten sind. Es besteht jedoch die Gefahr, dass die angestoßenen \\ Reformen auf halbem Weg steckenbleiben und auf diese Weise zu einer Verstärkung \\ von Bildungs- und sozialer Ungleichheit beitragen, statt diese abzubauen. Diese \\ These wird im Folgenden anhand einer kritischen Diskussion der Entwicklungen \\ in den Bereichen frühkindliche Erziehung, Schulpolitik und berufliche Bildung \\ entwickelt und diskutiert. Die Auswirkungen der digitalen Transformation \\ der Arbeitswelt werden hierbei in besonderer Weise berücksichtigt.
}

MARIUS R. BUSEMEYER

\section{Einleitung}

Im Vergleich zu anderen Feldern der Sozialpolitik nimmt die Bildungspolitik eine gewisse Sonderstellung ein, auch wenn sie zunehmend als integraler Bestandteil wohlfahrtsstaatlicher Regime betrachtet und analysiert wird (Busemeyer 2015; Solga 2014). Dies hat mehrere Gründe: Erstens nimmt der Stellenwert von Bildung im Zuge der strukturellen Transformation der industriellen Ökonomien hin $\mathrm{zu}$ dienstleistungsorientierten Wissensgesellschaften kontinuierlich zu. Zweitens stoßen die klassischen Instrumente der Sozialpolitik zunehmend an fiskalische und politische Grenzen. Die Umsetzung einer nachfrageorientierten Wirtschaftspolitik erscheint in Zeiten wirtschaftlicher Globalisierung wenig aussichtsreich. Stattdessen könnte eine inklusive Bildungspolitik auf eine Verbesserung der Angebotsseite der Arbeitsmärkte zielen. Das Rezept zur Vermeidung von sozialer Ungleichheit auf dem Arbeitsmarkt wäre dann, durch eine möglichst gerechte und breite Verteilung von Fertigkeiten (skills) der Entstehung von Lohnungleichheit entgegenzuwirken.

Aus diesen Gründen hat die Bildungspolitik einen zentralen Stellwert in der Debatte um die Transformation europäischer Wohlfahrtsstaaten in Richtung eines "Sozialinvestitionsmodells“ (Garritzmann et al. 2017; Hemerijck 2017; Morel et al. 2012). Die zentrale These der Befürworter des Sozialinvestitionsstaates ist, dass Sozialpolitik möglichst früh in individuelle Bildungs- und Arbeitsmarktkarrieren eingreifen müsse, um so die Entstehung von Ungleichheit möglichst effektiv und effizient $\mathrm{zu}$ vermeiden (Esping-Andersen 2002) statt diese - wie es mit den Instrumenten der klassischen Sozialpolitik geschieht - nachträglich zu bekämpfen. Gezielte Investitionen in Bildung können klassenbezogene Ungleichheiten, aber auch Ungleichheiten zwischen Geschlechtern, effektiv beseitigen, etwa wenn durch den Ausbau von frühkindlicher Bildung die Erwerbsbeteiligung von Frauen gefördert wird. Gleichzeitig sollte davor gewarnt werden, Bildungspolitik als „Wunderwaffe“ zu betrachten, mit deren Hilfe alle Probleme der Sozialpolitik gelöst werden können. Daher sprechen sich viele prinzipielle Befürworter des Sozialinvestitionsmodells dafür aus, soziale Investitionen als Ergänzung zu den bestehenden klassischen Instrumenten der Sozialpolitik auszubauen, statt diese zu ersetzen (Allmendinger/Nikolai 2010).

Dass Bildungspolitik nicht die Funktion einer „Wunderwaffe " übernehmen kann, liegt auch daran, dass sie sich mit einer Reihe von neuen Herausforderungen konfrontiert sieht. Bei der folgenden Diskussion dieser Herausforderungen möchte ich auf Tendenzen aufmerksam machen, die in der aktuellen Bildungspolitik erkennbar sind, sich aber noch nicht zwangsläufig konkretisiert haben. Ich beziehe mich dabei primär auf den Fall Deutschland, ergänzt um Verweise auf den internationalen Vergleich. 
Meine zentrale These ist, dass sich Bildungspolitik - vor allem aus Sicht der Gewerkschaften und der Sozialdemokratie - mit einer Reihe von Dilemmata konfrontiert sieht: Politische Ansätze und Instrumente, die auf den Abbau von Ungleichheiten abzielen, können zur Entstehung neuer Ungleichheiten beitragen. Ich diskutiere diese These im Folgenden anhand einer kritischen Reflexion der wichtigsten Reformen in den Bereichen frühkindliche Erziehung und Bildung, Schulpolitik und berufliche Bildung. Der Sektor der Hochschulpolitik muss als Platzgründen leider unberücksichtigt bleiben, obwohl dieser Sektor natürlich ebenfalls eine wichtige Bedeutung im Hinblick auf soziale Mobilität hat.

\section{Alte und neue Ungleichheiten im Ausbau frühkindlicher Bildung und Erziehung}

Der Sektor der frühkindlichen Bildung und Erziehung, insbesondere für Kleinkinder unter drei Jahren, ist in den letzten Jahren in Deutschland massiv ausgebaut worden. So hat die Zahl der Kinder im Alter von unter drei Jahren, die eine Tagesbetreuung außer Haus in Anspruch nehmen (über Tagespflegepersonen oder in Kitas), von $361623 \mathrm{im}$ Jahr 2008 auf $660750 \mathrm{im} \mathrm{Jahr} 2014$ zugenommen, was einer gesamtdeutschen Betreuungsquote von 32,3\% entspricht (BMFSFJ 2015). Dieser massive Ausbau von Betreuungsmöglichkeiten ist auch im internationalen Vergleich bemerkenswert (Fleckenstein et al. 2011; Morgan 2013).

Aus einer Perspektive der sozialen Gerechtigkeit bringt der Ausbau von Betreuungsmöglichkeiten für Kleinkinder positive Effekte. Zum einen, wie bereits oben erwähnt, konstatiert die einschlägige Forschung (Esping-Andersen 2002; Heckman 2006), dass Interventionen zu einem frühen Zeitpunkt von Bildungskarrieren besonders effektiv seien, um spätere Ungleichheiten zu vermeiden. Die Kleinkindphase ist demnach besonders wichtig für die intellektuelle, soziale und persönliche Entwicklung von Kindern, sodass eine umfassende Versorgung von Kleinkindern mit hochqualitativer Betreuung hier langfristig positive Effekte haben kann. Zum zweiten hat der Ausbau von Kinderbetreuungsmöglichkeiten positive Auswirkungen auf die Erwerbschancen junger Eltern. Insbesondere junge Mütter, die im Vergleich zu Vätern de facto weiterhin den größeren Teil der Erziehungsaufgaben übernehmen, profitieren von einer gut ausgebauten Betreuungsinfrastruktur, denn diese erleichtert die Vereinbarkeit von Familie und Beruf. Somit stärkt ein Ausbau der frühkindlichen Erziehung die finanziellen Grundlagen des Sozialstaates und mindert geschlechterbedingte Ungleichheiten.

Dieser kurze Überblick legt nahe, dass der Ausbau von frühkindlicher Bildung und Erziehung einen wichtigen
Beitrag zur Förderung von sozialer Gerechtigkeit leisten kann. Zudem sind Investitionen in diesen Bildungsbereich beliebt und - im Vergleich zu anderen Sozialpolitiken - verhältnismäßig kostengünstig (Bonoli 2013, S. 8). In Deutschland wird der Ausbau dieses Bereichs häufig auf eine engagierte Politik der ehemaligen Familienministerin und heutigen Verteidigungsministerin Ursula von der Leyen (CDU) zurückgeführt (Fleckenstein et al. 2011; Morgan 2013). Detaillierte Analysen der Staatstätigkeiten auf den unteren Regierungsebenen zeigen allerdings, dass auch dort wichtige Entscheidungen getroffen werden. Die Bundesebene setzt zwar wesentliche Rahmenbedingungen, wie etwa die Einführung eines Rechtsanspruchs auf einen Betreuungsplatz für Unter-Dreijährige im Jahr 2013 oder die Bereitstellung von finanziellen Mitteln zum Ausbau der Infrastruktur. Die Länderebene bleibt jedoch wichtig, da die Länder die Bundesmittel durch eigene Investitionen ergänzen (Andronescu/Carnes 2015; Busemeyer/ Seitzl 2017).

Trotz des dominanten Expansionstrends der letzten Jahre zeichnet sich jedoch eine neue Herausforderung ab: Solange der Ausbau der frühkindlichen Bildung und Erziehung nicht von einem Großteil der adressierten Eltern wahrgenommen wird, besteht die Gefahr, dass bestehende Ungleichheiten nicht vermindert, sondern verstärkt werden. Auch wenn die Betreuungsquote für Kinder unter drei Jahren in den letzten Jahren stetig angewachsen ist, scheint sich die Dynamik in den letzten Jahren etwas abgeschwächt zu haben: Die gesamtdeutsche Betreuungsquote für das Jahr 2017 liegt bei $33,1 \%,{ }^{1}$ und damit in etwa auf dem Niveau von 2014 (siehe oben), nachdem sie in den Jahren zuvor stark angestiegen war. Zwar bestehen laut dem Fünften Bericht zur Evaluation des Kinderförderungsgesetzes der Bundesregierung - weiterhin signifikante Differenzen zwischen dem Betreuungsbedarf und der tatsächlichen Betreuungsquote (BMFSFJ 2015, S.2), vor allem in den westdeutschen Bundesländern. Dennoch liegt dieser Analyse zufolge der geschätzte Betreuungsbedarf in den westdeutschen Bundesländern bei unter $40 \%$ (in den ostdeutschen Ländern liegt er etwa 20 Prozentpunkte darüber). Dies bedeutet, dass - was die tatsächliche Betreuung von Kleinkindern angeht - ca. zwei Drittel der Kinder unter drei Jahren nicht an einer außerhäuslichen Form der Kinderbetreuung teilnehmen und dass auch der geschätzte Betreuungsbedarf nicht darauf hindeutet, dass dieser Anteil mittelfristig deutschlandweit auf über $50 \%$ steigen wird - trotz Rechtsanspruch auf einen Betreuungsplatz.

Diese Zahlen sind bedeutsam, denn Analysen zu Beteiligungsmustern in der frühkindlichen Bildung und Er-

\footnotetext{
https://www.destatis.de/DE/ZahlenFakten/Gesellschaft Staat/Soziales/Sozialleistungen/Kindertagesbetreuung/ Tabellen/Tabellen_Betreuungsquote.html (abgerufen am 25. März 2018)
} 
ziehung haben gezeigt, dass Eltern aus den höheren Bildungs- und Einkommensschichten ihre Kinder eher in außerhäusliche Formen der Betreuung geben als Eltern aus unteren Einkommensschichten (van Lancker 2013; Pavolini/van Lancker 2018). Solange ein Großteil der Bevölkerung Kleinkinder nicht in eine außerhäusliche Form der Betreuung gibt, profitieren von deren Ausbau vor allen Dingen die gut gebildeten Mittelschichten, und zwar auf zweierlei Weise: Zum anderen wird die Entwicklung von Kindern - falls die Qualität der Betreuung gewährleistet ist - in Kinderbetreuungseinrichtungen aktiv gefördert, während sich Defizite der Kinder aus bildungsfernen Schichten akkumulieren. Zum anderen können Eltern, deren Kleinkinder außerhäuslich betreut werden, ihre Berufstätigkeit schneller wieder aufnehmen und damit als Doppelverdiener-Haushalte den Abstand zu den Einkommensschwachen weiter vergrößern. Beide Faktoren können bestehende Bildungs- und Einkommensungleichheiten verstärken, statt diese abzubauen.

Zur Lösung dieses Dilemmas gibt es keine einfachen Antworten. Die Politik hat durch die Schaffung eines Rechtsanspruchs auf einen Betreuungsplatz bereits sehr weitreichende und wichtige Grundlagen geschaffen. Bislang wird dieser Rechtsanspruch aber nur, wie angesprochen, von einer relativen Minderheit genutzt bzw. eingefordert. Dies wird dann zum Problem, wenn gerade Eltern aus bildungs- und einkommensschwachen Schichten diejenigen sind, die den Rechtsanspruch auf einen Kinderbetreuungsplatz nicht wahrnehmen. Deswegen erscheint es notwendig, dass die Politik mittelfristig auch einen Wandel der Betreuungskultur fördert, damit mehr Eltern ihren Rechtsanspruch auf Betreuung - zusätzlich auch motiviert durch eine verbesserte Qualität des Angebotes - einlösen. Eine konkrete Maßnahme in diesem Zusammenhang wäre zum Beispiel, durch eine Anpassung des Steuer- und Abgabensystems gezielt finanzielle Anreize zu setzen. Auch die institutionelle Ausgestaltung des Betreuungsangebots spielt eine entscheidende Rolle. Beispielsweise könnten die Öffnungszeiten von Kitas signifikant ausgeweitet werden, sodass auch Personen, die in Schichtarbeit arbeiten, diese in Anspruch nehmen können. Auch die hohen Kita-Gebühren können für manche - trotz Einkommensabhängigkeit und Sonderregelungen für Geringverdiener - eine faktische Hürde bleiben. Hinzu kommen weiterhin große regionale Ungleichheiten hinsichtlich der Versorgung, also der Verfügbarkeit von Kita-Plätzen, sowie partielle Qualitätsdefizite (Bertelsmann Stiftung 2014).

Ohne Frage: Die Behebung dieser Defizite kostet Geld. Im internationalen Vergleich der öffentlichen Ausgaben für frühkindliche Erziehung liegt Deutschland - trotz des Ausbaus der letzten Jahre - aber noch viel weiter zurück als in anderen Bereichen: Laut den neuesten verfügbaren Zahlen (für das Jahr 2013) der OECD betragen die Ausgaben für frühkindliche Erziehung in Deutschland lediglich $0,2 \%$ des Bruttoinlandsprodukts im Vergleich zu 1,1\% in Schweden und immerhin $0,6 \%$ in Frankreich. ${ }^{2}$ Im Ver- gleich zu den Budgets in den klassischen Bereichen der Sozialpolitik (Rente, Gesundheit, Arbeitsmarkt) sind dies immer noch kleine Größen.

Der entscheidende Punkt ist jedenfalls, dass der Ausbau der frühkindlichen Erziehung und Bildung nicht auf halbem Weg steckenbleiben darf, sondern engagiert und konsequent fortgesetzt werden muss, wenn er langfristig $\mathrm{zu}$ einer Verminderung von sozialer Ungleichheit beitragen soll. Die Ausbaudynamik der letzten Jahre ist ganz wesentlich von der großen Übernachfrage nach Betreuungsplätzen getrieben worden. Für die nächsten Jahre ist mittelfristig zu erwarten, dass diese starke Übernachfrage zurückgeht. Die politische Herausforderung besteht dann darin, die Ausbaupolitik trotz konstant knapper Haushalte und konkurrierender Ansprüche beständig fortzuführen. Eine dauerhafte Konsolidierung des Systems auf einem Niveau, auf dem etwa die Hälfte der Elternschaft die Betreuungsangebote nicht annimmt, wird nicht effektiv zu einer Reduzierung von Ungleichheit beitragen. Der notwendige Wandel der Betreuungskultur wird dann wahrscheinlicher, wenn ein konsequenter Ausbau der Angebotsinfrastruktur den Kulturwandel aktiv befördert - dies wäre ein wichtiger Unterschied zu der reaktiven, auf die Beseitigung akuter Defizite gerichteten Politik der letzten Jahre.

\section{Schulpolitik: Alte und neue Formen der Stratifizierung}

Diskussionen um die Reform von Schulstrukturen auf der Sekundarschulebene haben seit Jahrzehnten die Dynamik bildungspolitischer Debatten in Deutschland beherrscht (Busemeyer 2015; Helbig/Nikolai 2015). Hierbei haben sich linke Parteien und Gewerkschaften tendenziell als Verfechter des Gesamtschulprinzips (in verschiedenen Spielarten) profiliert und sich dafür ausgesprochen, die frühe Sortierung von Schülerinnen und Schülern auf unterschiedliche Bildungswege im Sekundarschulbereich abzuschaffen. Auf diese Weise - so das Argument - könnten Bildungschancen für Kinder aus sozial schwachen Familien verbessert werden, denn gegliederte Schulsysteme mit vielen „Entscheidungspunkten“ führten dazu, dass der familiäre Hintergrund einen größeren Einfluss auf Bildungsentscheidungen bekomme als in Gesamtschulsystemen (dazu stellvertretend für viele: Pfeffer 2008; Powell/Solga 2011; Schlicht et al. 2010). Bereits in den 1970er Jahren wurden Debatten zur Reform von Schulstrukturen geführt, die - in Abhängigkeit von der partei-

2 http://www.oecd.org/els/family/database.htm\#public_policy (abgerufen am 25. März 2018) 
politischen Färbung der Landesregierung - eine große Vielfalt von Bildungssystemen im Bundesländervergleich hervorgebracht haben, die sich auf unterschiedliche Weise auf Bildungsungleichheit auswirken können (Helbig/Nikolai 2015). Der „PISA-Schock“ Anfang der 2oooer Jahre hat eine neue Welle von Bildungsreformen ausgelöst und damit indirekt und mit Langzeitwirkung auch die Schulstruktur-Debatte wiederbelebt. Hinzu kommen demografisch bedingte Veränderungen, insbesondere zurückgehende Schülerzahlen in ländlichen Regionen, sowie das Schulwahlverhalten von Eltern- und Schülerschaft, die mittlerweile die Einschulung in eine Hauptschule zu vermeiden suchen, was zu einem graduellen Niedergang dieser Schulform geführt hat.

Die Grundtendenz der Schulstrukturreformen der letzten Jahre war eine graduelle Überwindung des gegliederten Sekundarschulsystems und eine graduell stärkere Durchsetzung des Prinzips des „Gemeinsamen Lernens“. Der zentrale Unterschied zwischen den heutigen Reformdebatten und den ambitionierteren Gesamtschulreformen der 1970er Jahren ist, dass die dauerhafte Existenz des Gymnasiums als eigenständige und unabhängige Schulform nicht mehr grundsätzlich infrage gestellt wird. Stattdessen zeichnet sich ein Zwei-Säulen-Modell als Kompromiss zwischen den Befürwortern und Skeptikern des Gesamtschulprinzips ab. Es ist somit eine Frage der Perspektive, ob dieser Kompromiss eher ein halb volles oder halb leeres Glas im Hinblick auf die Durchsetzung des Ziels des „Gemeinsamen Lernens“ darstellt.

Im Zwei-Säulen-Modell bleibt zum einen das Gymnasium als eigenständige Schulform erhalten. Zum anderen werden in der zweiten Säule des Modells verschiedene bestehende Schularten zu neuen Schultypen fusioniert. Dies betrifft insbesondere Haupt- und Realschulen, aber - je nach Bundesland - auch andere vormals existierende, teilintegrative Schulformen. Natürlich unterscheiden sich die Modelle im Detail je nach Bundesland, ebenso wie die Bezeichnungen der neuen Schultypen. Dennoch bleibt festzuhalten, dass das klassische dreigliedrige Sekundarschulsystem in Reinform inzwischen nur noch in Bayern existiert. Sachsen - am anderen Ende des Spektrums - hielt nach der Wiedervereinigung als einziges ostdeutsches Bundesland am Zwei-Säulen-Modell fest und tut dies auch heute noch. Hinzu kommt, dass einige Bundesländer (insbesondere Baden-Württemberg und Nordrhein-Westfalen) das berufliche Abitur als Alternative zum rein gymnasialen Abitur deutlich ausgeweitet haben, was die Durchlässigkeit zwischen dem beruflichen und dem akademischen Sektor langfristig verbessern dürfte.

Aus der Perspektive der sozialen Gerechtigkeit sind die jüngsten Strukturreformen begrüßenswert, auch wenn sie sicherlich ambitionierter sein könnten. Die PISA-Debatte der 2oooer Jahre hat Politik und die Öffentlichkeit auf das hohe Maß an Bildungsungleichheit in Deutschland aufmerksam gemacht. Der Schluss, dass hierbei auch die Schulstrukturen eine Rolle spielen, liegt durch den Ver- weis auf die PISA-Gewinnerländer im Norden Europas nahe, die im Unterschied zu Deutschland wesentlich früher und entschiedener Gesamtschulstrukturen eingeführt haben. Insofern können Schulstrukturreformen durchaus ein effektiver Hebel zur Verminderung von Bildungsungleichheit und damit langfristig auch sozialer Ungleichheit sein.

Wie schon anhand des Beispiels der frühkindlichen Erziehung und Bildung gezeigt, ergeben sich allerdings auch im Kontext der Schulstrukturreformen neue Herausforderungen, die die Gefahr beinhalten, dass neue Ungleichheiten entstehen. Ähnlich wie im ersten Beispiel besteht eine erste Herausforderung darin, die Reformdynamik nicht auf halber Strecke zu verlieren. Gegenwärtig haben die Strukturreformen der letzten Jahre trotz des sich abzeichnenden Kompromisses des Zwei-Säulen-Modells nicht zu einer Konvergenz der Schulstrukturen zwischen den Bundesländern beigetragen, sondern im Gegenteil die Fragmentierung des gesamtdeutschen Bildungssystems massiv vergrößert (Tillmann 2016).

So haben einige Bundesländer (z. B. Baden-Württemberg oder Nordrhein-Westfalen) die neuen teilintegrierten Schulformen zusätzlich zu den bestehenden Schulstrukturen eingeführt, sodass hier nun vier oder sogar noch mehr Schulformen auf der Sekundarschulebene existieren. Andere sind konsequent auf ein zweigliedriges System umgestiegen (außer dem oben erwähnten Sachsen z. B. noch Hamburg und das Saarland). Dieses aus gesamtdeutscher Perspektive fragmentierte Schulsystem erscheint nicht nur intransparent und wenig effizient, es birgt auch die Gefahr der Entstehung neuer Ungleichheiten. Je stärker sich die Schullandschaft ausdifferenziert, desto ungleicher werden die Bildungs- und Ausbildungsbedingungen für Kinder und Jugendliche in unterschiedlichen Bundesländern, selbst wenn die Schulreformen innerhalb der Bundesländer zunehmend das Prinzip des "Gemeinsamen Lernens“ umsetzen.

Eine zweite Herausforderung für die Bildungspolitik ergibt sich indirekt aus den Strukturreformen: Diese Reformen - in Verbindung mit anderen Entwicklungen wie der Zunahme der Zahl von Flüchtlingskindern in Schulen und der konsequenteren Umsetzung des Inklusionsprinzips an Schulen - haben dazu beigetragen, dass die Unterschiede in der sozio-kulturellen Zusammensetzung der Schülerschaften zwischen Schulen und Schulformen tendenziell abnehmen, dafür aber die Heterogenität innerhalb von Schulen und einzelnen Klassenverbünden zunimmt. Da die Lehrerausbildung in Deutschland allerdings weithin die Logik des gegliederten Schulsystems widerspiegelt, sind Lehrerinnen und Lehrer auf die Anforderungen, die eine zunehmende Heterogenität der Schülerschaft innerhalb des Klassenverbundes an sie stellt, häufig nur unzureichend vorbereitet. Dies zeigte sich in zugespitzter Form bei der Umsetzung des Inklusionsprinzips an Schulen (Blanck et al. 2014). Manche Bundesländer haben dies umgesetzt, indem sie Eltern von Kindern 
mit Förderbedarf ein Wahlrecht auf Beschulung in regulären oder Förderschulen einräumten. Die daraus resultierenden Schwierigkeiten in der Umsetzung haben zu Widerstand aufseiten der Lehrer- und Elternschaft geführt, der zumindest im Fall Nordrhein-Westfalen zur Abwahl der rot-grünen Landesregierung im Jahr 2017 beigetragen haben dürfte.

Die Erkenntnis aus diesem und ähnlichen Fällen sollte jedoch nicht sein, dass inklusive Bildungsreformen an sich der falsche Weg sind, sondern dass die Bildungspolitik mehr tun muss, um die Schwierigkeiten, die der Umgang mit Heterogenität innerhalb von Schulen und Klassenverbünden mit sich bringt, zu bewältigen. Dies beinhaltet zum einen eine Neuausrichtung der Lehrerausbildung, die mehr Wert auf die Vermittlung von inklusiven Lehrinhalten und -methoden legen sollte. Zum anderen sollte die Politik ausreichende und zusätzliche Ressourcen zur Verfügung stellen, wie etwa Personalmittel zur Umsetzung des „Zwei-Lehrer-Prinzips“ sowie zum Ausbau der sozialen und psychologischen Betreuungskapazitäten, damit sich Schulen, Lehrerinnen und Lehrer den neuen Anforderungen gewachsen fühlen.

\section{Die Zukunft der Berufsbildung vor dem Hintergrund der Digitalisierung und Akademisierung}

In einem dritten Beispiel möchte ich auf neue Herausforderungen im Bereich der beruflichen Bildung eingehen. Das deutsche Berufsbildungssystem - insbesondere das duale System der betrieblichen Ausbildung als sein Herzstück - erfreut sich hierzulande sowie international einer hohen Wertschätzung und Beliebtheit. Die Stärken der dualen Ausbildung - gut ausgebildete Fachkräfte für die Unternehmen, niedrige Jugendarbeitslosenquoten und einfache Übergänge von der Ausbildung in den Beruf sind weithin bekannt. Busemeyer (2015) und Solga (2014) haben außerdem gezeigt, dass sozio-ökonomische Ungleichheit in Ländern mit einem gut ausgebauten Berufsbildungswesen geringer ist als in anderen Ländern.

Doch trotz der offiziell dauerhaft niedrigen Jugendarbeitslosenquote leidet das Ausbildungssystem seit Jahren an gewissen strukturellen Ungleichgewichten, die mit der Marktabhängigkeit des Ausbildungsangebots zu tun haben. Besonders zu Beginn der 2oooer Jahre bis zur Mitte dieses Jahrzehntes klaffte eine große Lücke zwischen der starken Nachfrage von Jugendlichen nach Ausbildungsplätzen und dem quantitativ nicht ausreichenden Angebot der Unternehmen. Die Folge war, dass immer mehr Jugendliche in Alternativen zur regulären Ausbildung im sogenannten Übergangssektor vermittelt wurden (Baethge et al. 2007).
Die Situation auf dem Ausbildungsmarkt hat sich in den letzten Jahren jedoch stetig verbessert. Das zahlenmäßige Verhältnis zwischen der Zahl der angebotenen Ausbildungsstellen und der bei Arbeitsagenturen und Jobcentern gemeldeten Ausbildungsbewerberinnen und -bewerber (die Angebots-Nachfrage-Relation, ANR) hat sich seit 2009 von 100,4 auf 104,2 im Jahr 2016 zugunsten der Nachfrageseite verschoben (BMBF 2017, S. 21). ${ }^{3}$ Diese Zahlen berücksichtigen jedoch nicht diejenigen Jugendlichen, die trotz Ausbildungswunsch in eine Alternative eingemündet sind. Bei Berücksichtigung dieser Tatsache zeigt sich ein deutlich ungünstigeres Bild, aber auch aus dieser Perspektive sind in den letzten Jahren Verbesserungen der Marktlage zu beobachten (ebd., S. 21). Im gleichen Zeitraum ist die Zahl der unbesetzten Ausbildungsstellen von 17564 auf 43479 deutlich angestiegen und entspricht etwa $10 \%$ der im Jahr 2016 begonnenen betrieblichen Ausbildungen (ebd., S. 20). Des Weiteren ist die Zahl der Jugendlichen, die in Form von teilqualifizierender Ausund Weiterbildung (zunächst) in den Übergangssektor einmünden, deutlich von einem Höhepunkt im Jahr 2005 (417 649 Jugendliche) auf ein Tief im Jahr 2014 (252 670 Jugendliche) zurückgegangen. In den letzten beiden Jahren ist wieder ein Anstieg auf 298781 Jugendliche im Jahr 2016 zu beobachten, was allerdings mit der Integration von jugendlichen Flüchtlingen und Zugewanderten zu tun haben könnte (ebd., S. 42). Ins Bild passen auch zunehmende Schwierigkeiten der Betriebe, ihre Ausbildungsplätze zu besetzen (ebd., S. 66f.). Dies gilt insbesondere für kleine Betriebe und das Handwerk mit der Folge sinkender Ausbildungsbeteiligung in diesen Sektoren.

Die generell positiven Entwicklungen auf dem Ausbildungsstellenmarkt können allerdings nicht über die Tatsache hinwegtäuschen, dass die strukturellen Probleme und Schwächen des Systems weiterhin bestehen. Im Gegenteil: Die Verbesserung der Ausbildungsstellensituation vermindert den Problemdruck und damit auch die Notwendigkeit, grundlegende Reformen anzugehen. Die rot-grüne Bundesregierung (und die erste Große Koalition unter Kanzlerin Merkel als ihre Nachfolgerin) hatte zur Bekämpfung der damaligen Krise auf dem Ausbildungsstellenmarkt vor allen Dingen darauf gesetzt, die Beteiligung an Ausbildung für Arbeitgeber attraktiver zu machen (Busemeyer 2009). Beispiele hierfür waren die Wiedereinführung zweijähriger Ausbildungsberufe, Flexibilisierung und Differenzierung von Ausbildungsinhalten und -verläufen, die zeitweise Aussetzung der Ausbilder-Eignungsverordnung sowie der Abschluss eines Ausbildungspaktes zwischen Regierung und Wirtschaftsvertretern, an dem die Gewerkschaften nicht beteiligt waren. Ob diese Maß-

3 Ein Wert von 100 signalisiert ein zahlenmäßiges Gleichgewicht zwischen Angebot und Nachfrage, ein Wert von über 100 somit ein rechnerisches „Überangebot" an Ausbildungsstellen. 
nahmen tatsächlich zu den jüngsten Verbesserungen auf dem Ausbildungsmarkt beigetragen haben, ist fraglich und empirisch schwer zu überprüfen. Nach einem kurzen, aber deutlichen Anstieg der Zahl der von Unternehmen angeboten Ausbildungsstellen in den Jahren 2006 bis 2008 liegt das Niveau der von Arbeitgeberseite angebotenen Stellen im „Boomjahr“ 2016 jedenfalls wieder ziemlich genau auf dem Niveau des „Krisenjahres“ 2005. Dass die heutigen Verhältnisse dennoch ganz andere sind, liegt daher wesentlich an dem Rückgang der Nachfrage nach Ausbildungsplätzen, der - wie oben angesprochen - vor allen Dingen auf demografische Veränderungen, aber potenziell auch auf geändertes Wahlverhalten von Eltern und Jugendlichen zurückzuführen ist.

Gleichzeitig zeichnen sich neue Herausforderungen für die berufliche Bildung ab, die - wie in den vorangehenden Beispielen - häufig als negative Nebenfolgen vorangehender Politiken betrachtet werden können. Eine erste Herausforderung besteht in den sich abzeichnenden regionalen und berufsbezogenen Disparitäten hinsichtlich des Verhältnisses zwischen Angebot und Nachfrage auf dem Ausbildungsmarkt (BMBF 2017, S.7off.). Entgegen naiver Vorstellungen, dass sich in Zeiten zurückgehender Schülerzahlen die Nachfrageprobleme auf dem Ausbildungsstellenmarkt quasi von allein lösen, zeigen die jüngsten Entwicklungen, dass es sehr wohl eine Gleichzeitigkeit von Ausbildungsplatz- und Fachkräftemangel geben kann. Diese Passungsprobleme können zurückgehen auf

- regionale Unterschiede in Ausbildungsangebot und -nachfrage, die stark durch die lokalen Arbeitsmarktverhältnisse bedingt sind;

- berufsbezogene Unterschiede, die die relative Beliebtheit bzw. Unbeliebtheit von Ausbildungsberufen widerspiegeln;

- ein sich änderndes Wahlverhalten der besser qualifizierten Jugendlichen, die zunehmend der beruflichen Bildung den Rücken kehren und sich für ein Hochschulstudium entscheiden.

Passungsprobleme können zur Verfestigung von Ungleichheiten zwischen ökonomisch starken und schwachen Regionen führen, aber auch geschlechterbezogene Ungleichheiten bestärken, da junge Frauen und Männer sich weiterhin systematisch in ihren Berufswahlentscheidungen voneinander unterscheiden.

Politisch gesprochen besteht die Aufgabe darin, diese Passungsprobleme genauso ernst zu nehmen wie die Krisen des Ausbildungsmarktes zuvor. Passungsprobleme deuten nämlich auch sich verfestigende Ungleichheitslagen in bestimmten Regionen und/oder Schichten an, sodass die Betroffenen durch die allgemein positiven Entwicklungen auf dem Ausbildungsstellenmarkt - relativ gesprochen - weiter abgehängt werden. In dieses Bild passt auch die Tatsache, dass die Quote der 20- bis 34-Jährigen ohne Berufsabschluss seit Jahren bei ungefähr 13\% stagniert (ebd., S. 74).
Die zweite große Herausforderung für die berufliche Bildung stellt die fortschreitende Digitalisierung und Automatisierung der Arbeitswelt dar. Auch wenn die Berufsbildung entgegen den Erwartungen mancher Kritiker die Einführung neuer Informations- und Kommunikationstechnologien in den 1990er Jahren erfolgreich gemeistert hat, gibt es gute Gründe für die These, dass die gegenwärtige und zukünftige Phase des radikalen technologischen Wandels qualitativ anders und damit folgenreicher sein könnte. Das gleichzeitige Auftreten von technologischen Veränderungen in unterschiedlichen Bereichen wie der Robotik, der Künstlichen Intelligenz, Big Data sowie der Automatisierung und Vernetzung von Produktionssystemen könnte zu einem Wegfall einer beträchtlichen Zahl von Arbeitsplätzen führen. Die Spannbreite der einschlägigen Studien variiert dabei allerdings erheblich: Frey und Osborne (2017, S. 265) schätzen, dass $47 \%$ der Beschäftigungsverhältnisse in den USA einem hohen Automationsrisiko ausgesetzt sind, während Dengler und Matthes mit einer ähnlichen, aber leicht veränderten Methodik auf einen Wert von $15 \%$ für Deutschland kommen (Dengler/Matthes 2015, S.7). Die Forschung zu den bisherigen Auswirkungen des technologischen Wandels zeigt (Breemersch et al. 2017), dass sowohl einfache Berufe - vor allen Dingen personenbezogene und lokale Dienstleistungen - als auch anspruchsvolle Tätigkeiten, die ausgeprägte theoretische, organisatorische oder soziale Fertigkeiten erfordern, von den Auswirkungen der Automatisierung relativ geschützt sind. Die Berufe im mittleren Fachkräftesegment hingegen scheinen besonders negativ tangiert zu sein. Nach den Berechnungen von Dengler und Matthes sind Fachkraftberufe ( $45 \%$ der Beschäftigungsverhältnisse mit hohem Automationsrisiko) in gleicher Weise negativ von der nächsten Automatisierungswelle betroffen wie einfache Helferberufe, aber deutlich stärker als Expertenberufe (19\% Automatisierungsrisiko), die in der Regel einen Hochschulabschluss voraussetzen (Dengler/Matthes 2015, S. 12).

Die digitale Transformation der Arbeitswelt könnte somit zwei schon bestehende Entwicklungstendenzen massiv verstärken: eine Polarisierung von Arbeits- und Einkommenschancen sowie den Drang zur Akademisierung von Bildung und Ausbildung. Beide Trends bedingen und verstärken sich gegenseitig und setzen die berufliche Bildung unter Druck. Die Fliehkräfte im Berufsbildungssystem werden deswegen ebenfalls weiter zunehmen: Auf der einen Seite könnten Arbeitgeber zunehmend einfache Ausbildungsberufe einfordern oder gleich ganz auf die Ausbildung von Fachkräften verzichten und stattdessen angelernte Aushilfskräfte beschäftigen, wenn es sich um personenbezogene Dienstleistungen handelt (ein gutes Beispiel hierfür sind Nebentätigkeiten von Studierenden in der Gastronomie). Auf der anderen Seite wachsen die Ansprüche an die Berufsbildung am „oberen Ende“ der Skala: Die Attraktivität der Berufsbildung für Jugendliche mit guten Vorqualifikationen ist dann am ehesten gewähr- 
leistet, wenn sie gesicherte Perspektiven im Bereich der Hochschulbildung bieten kann.

Duale Studiengänge als Hybridlösung zwischen beruflicher Ausbildung und Hochschulstudium sind in den letzten Jahren stark ausgebaut worden - in Reaktion auf eine hohe Nachfrage sowohl vonseiten der Betriebe als auch der Jugendlichen (Graf 2018). Duale Studiengänge erhalten damit zwar einerseits die Attraktivität der beruflichen Bildung, tragen andererseits aber auch zu ihrer graduellen Unterminierung bei. Ein wichtiger Unterschied zwischen dualen Studiengängen und einer klassischen Ausbildung im dualen System ist nämlich, dass Arbeitgeber bei Ersteren sehr viel mehr Freiraum und Einfluss auf die Ausgestaltung der Inhalte der theoretischen Ausbildung haben (wie üblich: mit Unterschieden je nach Bundesland), da sie sich nicht wie in der dualen Ausbildung im Rahmen eines komplexen korporatistischen Aushandlungssystems mit anderen Akteuren arrangieren müssen. Die neue Gratwanderung besteht hier also darin, bei der Ausgestaltung von Ausbildungsinhalten die Interessen und Perspektiven der Bildungsteilnehmerinnen und -teilnehmer an einer breiten und nachhaltig qualifizierenden Berufsausbildung sicherzustellen und Ausbildungsgänge nicht zu stark auf einzelbetriebliche Bedürfnisse zuzuschneiden. Dies könnte dadurch erreicht werden, dass das „Berufsprinzip“ als normatives Leitbild auch in dualen Studiengängen stärker verankert wird. Zudem könnte der betriebliche Teil von dualen Studiengängen (wenn es nicht ohnehin schon regulierte Ausbildungsberufe sind) in den Regelungsbereich des Berufsbildungsgesetzes (BBiG) eingegliedert werden.

\section{Fazit}

In diesem kurzen Beitrag habe ich die - aus meiner Sicht - wichtigsten alten und neuen Herausforderungen, mit denen sich Bildungspolitik in Deutschland im Zeitalter der Digitalisierung und Globalisierung auseinandersetzen sollte, skizziert. In der Grundtendenz und aus der Perspektive der sozialen Gerechtigkeit sind viele Entwicklungen der letzten Jahre positiv zu bewerten. Auch wenn die Dinge sich langsamer und nicht immer entschieden in die "richtige“ Richtung bewegen - sie bewegen sich immerhin. Gleichzeitig habe ich eine Reihe von neuen Herausforderungen aufgezeigt, die häufig mit den negativen Nebenfolgen vorheriger Politiken zusammenhängen und Dilemma-Situationen erzeugen, wonach die Beseitigung alter Formen von Ungleichheit neue Ungleichheiten entstehen lässt.

Progressive Politik kann und sollte auf diese Situation auf zweierlei Weise reagieren: Zum einen sollte empirische Sozialforschung zur Entscheidungsfindung in stärkerer Weise herangezogen werden, um die komplexen Wechselwirkungen zwischen einzelnen Politiken und Politikfeldern besser zu verstehen. Durch eine Art sozialwissenschaftliches Frühwarnsystem könnte so der Entstehung neuer Ungleichheiten präventiv vorgebeugt werden. Die WSI-Mitteilungen haben sich in den letzten 70 Jahren bereits beispielhaft in der Vermittlung zwischen Wissenschaft und Praxis engagiert. Dennoch liegt Deutschland im Vergleich zu anderen Ländern bei der Umsetzung von „evidenzbasiertem Policy-Making“ noch zurück. Insofern bleibt die Förderung des Erkenntnistransfers zwischen Wissenschaft und Politik eine Daueraufgabe.
Zum anderen besteht aus einer eher politischen Perspektive die zentrale Aufgabe darin, die Ansprüche an und das Engagement für progressive Reformen weiterhin hoch zu halten. Die gegenwärtig guten Verhältnisse auf dem Ausbildungs- und Arbeitsmarkt sind zwar zu begrüßen, können aber dazu führen, dass der Reformeifer nachlässt, obwohl große Probleme im Bildungssystem weiterhin ungelöst sind. Schlimmer noch: Die Beispiele der frühkindlichen Erziehung und der Schulstrukturreformen haben gezeigt, dass Reformbemühungen, die auf halbem Weg stehenbleiben, Ungleichheiten sogar noch vergrößern können, statt diese zu bekämpfen. Insofern sollten die gegenwärtig guten wirtschaftlichen und fiskalpolitischen Bedingungen nicht Endpunkt, sondern Ausgangspunkt für eine anspruchsvolle und umfassende Reformagenda sein.

Die wichtigsten Handlungsfelder zur Umsetzung einer Bildungspolitik, die auf soziale Mobilität und Gerechtigkeit abzielt, sind:

(1) der weitere engagierte Ausbau von frühkindlicher Bildung und Erziehung, verbunden mit einem Perspektivenwechsel von einer eher quantitativen Expansion hin zu einer Verbesserung der Qualität der Betreuung;

(2) die Verbesserung der Durchlässigkeit zwischen und der Gleichwertigkeit von beruflicher und akademischer Bildung, beispielweise durch die engagierte Fortführung begonnener Strukturreformen und den weiteren Ausbau von hybriden Qualifikationen wie Berufsabitur und duale Studiengänge.

\section{LITERATUR}

Allmendinger, J./ Nikolai, R. (2010): Bildungs- und Sozialpolitik: Die zwei Seiten des Sozialstaats im internationalen Vergleich, in: Soziale Welt 61 (2), S. 105-119 Andronescu, C.G./ Carnes, M. E. (2015): Value coalitions and policy change: The impact of gendered patterns of work, religion and partisanship on childcare policy across German states, in: Journal of European Social Policy 25 (2),

S. 159-174

Baethge, M./ Solga, H./ Wieck, M. (2007): Berufsbildung im Umbruch. Signale eines überfälligen Aufbruchs, Friedrich-Ebert-Stiftung, Berlin

Bertelsmann Stiftung (2014): Öffentliche Anhörung zur Verbesserung des Ausbaus und der Qualität der Kindertagesstätten vom Ausschuss für Familie, Senioren, Frauen und Jugend, Deutscher Bundestag, Stellungnahme Kathrin Bock-Famulla, 10. November 2014, Berlin

Blanck, J. / Edelstein, B. / Powell, J. J.W. (2014): Auf dem Pfad zur inklusiven Bildung? Schulreformen in Deutschland und die UN-Behindertenrechtskonvention, in: Schuppener, S. / Bernhardt, N. / Hauser, M./ Poppe, F. (Hrsg.): Inklusion und Chancengleichheit: Diversity im Spiegel von Bildung und Didaktik, Bad Heilbrunn, S. 97-104

BMBF (Bundesministerium für Bildung und Forschung) (2017): Berufsbildungsbericht 2017, Bonn/Berlin BMFSFJ (Bundesministerium für Familie, Senioren, Frauen und Jugend) (2015): Fünfter Bericht zur Evaluation des Kinderförderungsgesetzes, Kurzfassung, Berlin

Bonoli, G. (2013): The origins of active social policy: Labour market and childcare policies in a comparative perspective, Oxford/New York

Breemersch, K./ Damijan, J. P./ Konings, J. (2017): Labour market polarization in advanced countries: Impact of global value chains, technology, import competition from China and labour market institutions, OECD Social, Employment and Migration Working Papers No. 197

Busemeyer, M. R. (2009): Wandel trotz Reformstau: Die Politik der beruflichen Bildung seit 1970, Frankfurt a. M.

Busemeyer, M.R. (2015): Skills and inequality: The political economy of education and training reforms in western welfare states, Cambridge/New York Busemeyer, M. R./ Seitzl, L. (2017): The partisan politics of early childhood education in the German Länder, in: Journal of Public Policy 38 (2), S. 243-274, https://doi.org/10.1017/S0143814X16000313 
Dengler, K./ Matthes, B. (2015): Folgen der Digitalisierung für die Arbeitswelt: Substituierbarkeitspotenziale von Berufen in Deutschland, Institut für Arbeitsmarkt- und Berufsforschung: IAB Forschungsbericht 11/2015

Esping-Andersen, G. (2002): A child-centred social investment strategy, in: Esping-Andersen, G. (Hrsg.): Why we need a new welfare state, Oxford/New York, S. 26-67

Fleckenstein, T. / Saunders, A. M. / Seeleib-Kaiser, M. (2011): The dual transformation of social protection and human capital: Comparing Britain and Germany, in: Comparative Political Studies 44 (12), S. 1622-1650

Frey, C. B./ Osborne, M. A. (2017): The future of employment: How susceptible are jobs to computerisation?, in: Technological Forecasting and Social Change 114 (C), S. 254-280

Garritzmann, J. L. / Häusermann, S. / Palier, B. / Zollinger, C. (2017): WOPSI:

The World Politics of Social Investment, Laboratory for Interdisciplinary Evaluation of Public Policies (LIEPP): LIEPP Working Paper 64, Paris

Graf, L. (2018): Combined modes of gradual change: The case of academic upgrading and declining collectivism in German skill formation, in: Socio-Economic Review 16 (1), S. 185-205

Heckman, J.J. (2006): Skill formation and the economics of investing in disadvantaged children, in: Science (312), S. 1900-1902

Helbig, M./ Nikolai, R. (2015): Die Unvergleichbaren: Der Wandel der Schulsysteme in den deutschen Bundesländern seit 1949, Bad Heilbrunn

Hemerijck, A. (Hrsg.) (2017): The uses of social investment, Oxford/New York van Lancker, W. (2013): Putting the child-centred investment strategy to the test: Evidence for the EU27, in: European Journal of Social Security 15 (1),

S. 4-27

Morel, N./ Palier, B./ Palme, J. (2012): Beyond the welfare state as we knew it? in: Morel, N. / Palier, B./ Palme, J. (Hrsg.): Towards a social investment welfare state? Ideas, policies and challenges, Bristol/Chicago, S. 1-30

Morgan, K.J. (2013): Path shifting of the welfare state: Electoral competition and the expansion of work-family policies in Western Europe, in: World Politics 65 (1), S. 73-115
Pavolini, E./ van Lancker, W. (2018): The Matthew effect in childcare Use: A matter of policies or preferences?, in: Journal of European Public Policy 25 (6), S. 878-893

Pfeffer, F. T. (2008): Persistent inequality in educational attainment and its institutional context, in: European Sociological Review 24 (5), S. 543-565

Powell, J.J. W./ Solga, H. (2011): Why are higher education participation rates in Germany so low? Institutional barriers to higher education expansion, in: Journal of Education and Work 24 (1-2), S. 49-68

Schlicht, R./ Stadelmann-Steffen, I. / Freitag, M. (2010): Educational inequality in the EU: The effectiveness of national education policy, in: European Union Politics 11 (1), S. 29-59

Solga, H. (2014): Education, economic inequality and the promises of the social investment state, in: Socio-Economic Review 12 (2), S. 269-297

Tillmann, K.-J. (2016): Das Sekundarschulsystem auf dem Weg in die Zweigliedrigkeit: Historische Linien und aktuelle Verwirrungen, Bundeszentrale für politische Bildung, http://www.bpb.de/gesellschaft/bildung/zukunft-bildung/215556/ zweigliedrigkeit? $p=$ all

\section{AUTOR}

MARIUS R. BUSEMEYER, Dr. habil., ist Professor für Politikwissenschaft, insbesondere Policy-Analyse und politische Theorie, an der Universität Konstanz. Forschungsschwerpunkte: Internationaler Vergleich von Bildungs- und Sozialpolitik, vergleichende politische Ökonomie sowie Analysen zur öffentlichen Meinung und Einstellungen zum Wohlfahrtsstaat.

arius.busemeyer@uni-konstanz.de 